\title{
THE CANONICAL COMPACTIFICATION OF A FINITE GROUP OF LIE TYPE
}

\author{
MOHAN S. PUTCHA AND LEX E. RENNER
}

\begin{abstract}
Let $G$ be a finite group of Lie type. We construct a finite monoid $\mathscr{M}$ having $G$ as the group of units. $\mathscr{M}$ has properties analogous to the canonical compactification of a reductive group. The complex representation theory of $\mathscr{M}$ yields Harish-Chandra's philosophy of cuspidal representations of $G$. The main purpose of this paper is to determine the irreducible modular representations of $\mathscr{M}$. We then show that all the irreducible modular representations of $G$ come (via the 1942 work of Clifford) from the one-dimensional representations of the maximal subgroups of $\mathscr{M}$. This yields a semigroup approach to the modular representation theory of $G$, via the full rank factorizations of the 'sandwich matrices' of $\mathscr{M}$. We then determine the irreducible modular representations of any finite monoid of Lie type.
\end{abstract}

\section{INTRODUCTION}

The purpose of this paper is to construct, for each finite group of Lie type, a certain monoid modeled on the canonical compactification of DeConcini and Procesi [6] for reductive groups. The natural way of accomplishing this is to start with a linear algebraic monoid $M$ with Frobenius endomorphism $\sigma: M \rightarrow M$ yielding a finite monoid $M_{\sigma}=\{x \in M \mid \sigma(x)=x\}$. This provides us with a highly structured procedure for constructing a large class of monoids of Lie type. The correct choice of $M$ then yields our canonical compactification $M_{\sigma}$. For the purpose of relating the modular and complex representation theories of $M_{\sigma}$ and its unit group $G$, we are naturally led to considering a universal central extension $\mathscr{M}$ of $M_{\sigma}$. We are able to construct this universal compactification $\mathscr{M}=\mathscr{M}(G)$ abstractly starting with any group $G$ of Lie type. For clarity in exposition we do this first, saving a discussion of the monoids $M_{\sigma}$ to the last section.

Using our universal monoid we count up the irreducible modular representations whose restriction to the unit group is irreducible. Using another counting method, we then find that any irreducible representation of $\mathscr{M}$ restricts to an irreducible representation of $G$. This essentially reduces the enumeration of irreducible modular representations of any finite monoid of Lie type to a combinatorial problem. Using the results of Clifford $[2,3]$, we are then able to show that the degree of a modular irreducible representation $\varphi$ of $G$ is equal to the rank of a certain matrix associated with $\varphi$ and $\mathscr{M}$. In fact the full rank

Received by the editors May 21, 1990 and, in revised form, February 10, 1991.

1980 Mathematics Subject Classification (1985 Revision). Primary 20G40, 20M30, 20 G05.

First author partially supported by NSF, second author partially supported by NSERC. 
factorization of the 'sandwich' matrix yields $\varphi$. In particular, we show that the modular Steinberg representation of $G$ has an idempotent separating extension to $\mathscr{M}$.

We also include a brief discussion of complex representations of $\mathscr{M}$ mainly for the purpose of pointing out that $\mathscr{M}$ also has natural connections with the complex representation theory of $G$. In particular, every irreducible representation of $G$ is a component of the restriction to $G$ of an irreducible cuspidal representation of $\mathscr{M}$, yielding a new interpretation of Harish-Chandra's philosophy of cuspidal representations.

\section{THE UNIVERSAL CANONICAL COMPACTIFICATION}

Let $M$ be a finite monoid with group of units $G$. If $X \subseteq M$, we let $E(X)=\left\{e \in X \mid e^{2}=e\right\} . M$ is regular if $a \in a M a$ for all $a \in M$. For $e \in E=E(M)$, the following three subgroups of $G$ are of critical importance in our work:

$$
\begin{aligned}
P & =P(e)=C_{G}^{r}(e)=\{x \in G \mid x e=e x e\}, \\
P^{-} & =P^{-}(e)=C_{G}^{l}(e)=\{x \in G \mid e x=e x e\}, \\
L & =L(e)=C_{G}(e)=\{x \in G \mid x e=e x\} .
\end{aligned}
$$

Let $G$ be a finite group of Lie type with Coxeter graph $\Gamma$, cf. [1]. For a parabolic subgroup $P$ of $G$, we let $R_{u}(P)$ denote its unipotent radical. By a (finite) monoid of Lie type on $G$, we mean a finite regular monoid $M$ with zero 0 and having group of units $G$ such that $M$ is generated by $E=E(M)$ and $G$, and

(1) For all $e \in E, P(e), P^{-}(e)$ are opposite parabolic subgroups of $G$ and for all $u \in R_{u}(P(e)), v \in R_{u}\left(P^{-}(e)\right), u e=e=e v$.

(2) For all $e, f \in E$, $e M=f M$ or $M e=M f$ implies $x^{-1} e x=f$ for some $x \in G$.

Monoids of Lie type were introduced in [11] with a different name (regular split monoids) as an abstraction of certain properties of linear algebraic monoids $[9,13,16]$. Section 4 and [17] provide examples of special monoids of Lie type.

Let $M$ be a monoid of Lie type on $G$. Then $G$ has a two-sided action on $M$. Let $\mathscr{U}$ denote the set of $G \times G$ orbits of $M$. These are the $\mathscr{J}$-classes of $M$ in the usual sense [4]. Define $G a G \geq G b G$ if $b \in M a M$. In this way $\mathscr{U}$ becomes a lattice. There is a cross section of idempotents of $e_{J}(J \in \mathscr{U})$ such that $J=G e_{J} G$ and for all $J_{1}, J_{2} \in \mathscr{U}, e_{J_{1}} e_{J_{2}}=e_{J_{2}} e_{J_{1}}=e_{J_{1} \wedge J_{2}} . \Lambda=\left\{e_{J} \mid J \in \mathscr{U}\right\}$ is called a cross section lattice. Moreover, $M=E(M) G$ and for all $J \in \mathscr{U}$,

$$
E(J)=\left\{x^{-1} e_{J} x \mid x \in G\right\} .
$$

There is a type map $\lambda: \mathscr{U} \rightarrow 2^{\Gamma}$ such that for all $J \in \mathscr{U}, P\left(e_{J}\right)=P_{\lambda(J)}, P^{-}\left(e_{J}\right)$ $=P_{\lambda(J)}^{-}$. We write $P_{J}, P_{J}^{-}, L_{J}, U_{J}, U_{J}^{-}$for $P_{\lambda(J)}, P_{\lambda(J)}^{-}, L_{\lambda(J)}, U_{\lambda(J)}, U_{\lambda(J)}^{-}$, respectively. We call $\lambda(J)$ the type of $J$ (and $e_{J}$ ), $\lambda$ the type of $M$. This type map completely determines the system of idempotents of $M$. For $J \in \mathscr{U}$, let

$$
K_{J}=\left\{x \in G \mid x e_{J}=e_{J} x=e_{J}\right\}
$$

Then $K_{J} \triangleleft L_{J}$. Let

$$
\mathscr{K}=\mathscr{K}(M)=\left\{\left(J, K_{J}\right) \mid J \in \mathscr{U}\right\}
$$


The type map $\lambda$ along with the data $\mathscr{K}(M)$, completely classifies a monoid of Lie type. We refer to $[11,12,13]$ for details.

Let $G$ be a group of Lie type with Coxeter graph $\Gamma, I \subseteq \Gamma$. Then $P_{I}^{-} P_{I}=$ $U_{I}^{-} L_{I} U_{I}$. We therefore have a natural map $\theta_{I}: P_{I}^{-} P_{I} \rightarrow L_{I} . \theta_{I}$ restricted to $P_{I}$ or $P_{I}^{-}$is a homomorphism. Moreover,

$$
\theta_{I}(a x)=a \theta_{I}(x), \quad \theta_{I}(x a)=\theta_{I}(x) a \text { for all } x \in P_{I}^{-} P_{I}, a \in L_{I} .
$$

We are now in a position to construct the universal compactification $\mathscr{M}=$ $\mathscr{M}(G)$. This is the universal monoid of Lie type with the properties of some very special monoids $M_{\sigma}$ that we will construct in Theorems 4.7 and 4.10. The monoids $M_{\sigma}$ are derived from linear algebraic monoids $M$ which are cones on the compactification of a reductive group. The importance of $\mathscr{M}$ will be clear in Sections 2 and 3.

Theorem 1.1. Let $G$ be a group of Lie type with Coxeter graph $\Gamma$. Start with idempotents $e_{I}(I \subseteq \Gamma)$. Let $J_{I}=G e_{I} G / \equiv, \mathscr{M}=\bigsqcup_{I \subseteq \Gamma} J_{I} \sqcup\{0\}$ where for $x, y, x_{1}, y_{1} \in G$, define $x e_{I} y \equiv x_{1} e_{I} y_{1}$ if $x_{1}^{-1} x \in P_{I}, y_{1} y^{-1} \in P_{I}^{-}$and $\theta_{I}\left(x_{1}^{-1} x\right)=\theta_{I}\left(y_{1} y^{-1}\right)$. For $a=x e_{I} y \in J_{I}, b=s e_{K} t \in J_{K}$, define

$$
a b= \begin{cases}x l e_{I \cap K} m t & \text { if } y s \in U_{I}^{-} l m U_{K}, l \in L_{I}, m \in L_{K}, \\ 0 & \text { if } y s \notin P_{I}^{-} P_{K} .\end{cases}
$$

Then $\mathscr{M}=\mathscr{M}(G)$ is a monoid of Lie type.

Proof. Since $\theta_{I}$ is a homomorphism when restricted to $P_{I}$ or $P_{I}^{-}$, we see that $\equiv$ is an equivalence relation. We next show that multiplication in $\mathscr{M}$ is welldefined. So let $a=x e_{I} y, a_{1}=x_{1} e_{I} y_{1} \in J_{I}, b=s e_{K} t, b_{1}=s_{1} e_{K} t_{1} \in J_{K}$ such that $a \equiv a_{1}$ and $b \equiv b_{1}$. Then

$$
\begin{array}{ll}
x_{1}^{-1} x \in P_{I}, & y_{1} y^{-1} \in P_{I}^{-}, \quad \theta_{I}\left(x_{1}^{-1} x\right)=\theta_{I}\left(y_{1} y^{-1}\right), \\
s_{1}^{-1} s \in P_{K}, & t_{1} t^{-1} \in P_{K}^{-}, \quad \theta_{K}\left(s_{1}^{-1} s\right)=\theta_{K}\left(t_{1} t^{-1}\right) .
\end{array}
$$

It follows that $y s \in P_{I}^{-} P_{K}$ if and only if $y_{1} s_{1} \in P_{I}^{-} P_{K}$. Let $y s, y_{1} s_{1} \in P_{I}^{-} P_{K}=$ $U_{I}^{-} L_{I} L_{K} U_{K}$. Then for some $l, l_{1} \in L_{I}, m, m_{1} \in L_{K}, y s \in U_{I}^{-} \operatorname{lm} U_{K}$ and $y_{1} s_{1} \in U_{I}^{-} l_{1} m_{1} U_{K}$. So $a b=x l e_{I \cap K} m t, a_{1} b_{1}=x_{1} l_{1} e_{I \cap K} m_{1} t_{1}$. Now since $x_{1}^{-1} x \in P_{I}$ and $U_{I} \triangleleft P_{I}$,

$$
\begin{aligned}
l_{1}^{-1} x_{1}^{-1} x l & \in U_{I} l_{1}^{-1} \theta_{I}\left(x_{1}^{-1} x\right) l=U_{I} l_{1}^{-1} \theta_{I}\left(y_{1} y^{-1}\right) l \\
& =U_{I} \theta_{I}\left(l_{1}^{-1} y_{1} y^{-1} l\right), \text { since } l, l_{1} \in L_{I} .
\end{aligned}
$$

Then

$$
\begin{aligned}
z & =l_{1}^{-1} y_{1} y^{-1} l \in l_{1}^{-1} U_{I}^{-} l_{1} m_{1} U_{K} s_{1}^{-1} s U_{K} m^{-1} l^{-1} U_{I}^{-} l \\
& =U_{I}^{-} m_{1} U_{K} s_{1}^{-1} s U_{K} m^{-1} U_{I}^{-} \\
& =U_{I}^{-} m_{1} s_{1}^{-1} s m^{-1} U_{K} U_{I}^{-}, \text {since } m, s_{1}^{-1} s \in P_{K}
\end{aligned}
$$

So for some $u_{1}, u_{2} \in U_{I}^{-}, v \in U_{K}$,

$$
z=u_{1} m_{1} s_{1}^{-1} s m^{-1} v u_{2} \text {. }
$$

Let $z_{1}=m_{1} s_{1}^{-1} s m^{-1} v$. Then since $z \in P_{I}^{-}$, we see that $z_{1} \in P_{I}^{-}$and $\theta_{I}(z)=$ $\theta_{I}\left(z_{1}\right)$. Since $s_{1}^{-1} s \in P_{K}$ we see that $z_{1}=m_{1} \theta_{K}\left(s_{1}^{-1} s\right) m^{-1} w$ for some $w \in$ $U_{K}$. Let $z_{2}=m_{1} \theta_{K}\left(s_{1}^{-1} s\right) m^{-1} \in L_{K}$. Then

$$
z_{1}=z_{2} w \in P_{K} \cap P_{I}^{-}=L_{I \cap K}\left(L_{K} \cap U_{I}^{-}\right)\left(U_{K} \cap P_{I}^{-}\right) .
$$


So $z_{2} \in L_{I \cap K}\left(L_{K} \cap U_{I}^{-}\right), w \in U_{K} \cap P_{I}^{-}$. Hence

$$
\theta_{I}(z)=\theta_{I}\left(z_{1}\right)=\theta_{I}\left(z_{2} w\right)=\theta_{I}\left(z_{2}\right) \theta_{I}(w), \quad \theta_{I}\left(z_{2}\right) \in L_{I \cap K}, \quad \theta_{I}(w) \in U_{K} \cap L_{I} .
$$

In particular, $\theta_{I}(z) \in P_{I} \cap P_{K}=P_{I \cap K}$. So

$$
l_{1}^{-1} x_{1}^{-1} x l \in U_{I} \theta_{I}(z) \subseteq U_{I} P_{I \cap K} \subseteq P_{I \cap K} .
$$

Moreover, since $U_{I} \cup U_{K} \subseteq U_{I \cap K}$,

$$
\begin{aligned}
\theta_{I \cap K}\left(l_{1}^{-1} x_{1}^{-1} x l\right) & =\theta_{I \cap K}\left(\theta_{I}(z)\right)=\theta_{I \cap K}\left(\theta_{I}\left(z_{2}\right) \theta_{I}(w)\right)=\theta_{I \cap K}\left(\theta_{I}\left(z_{2}\right)\right) \\
& =\theta_{I \cap K}\left(z_{2}\right), \text { since } U_{I}^{-} \subseteq U_{I \cap K}^{-} \\
& =\theta_{I \cap K}\left(m_{1} \theta_{K}\left(s_{1}^{-1} s\right) m^{-1}\right) .
\end{aligned}
$$

Similarly $m_{1} t_{1} t^{-1} m^{-1} \in P_{I \cap K}^{-}$. Now since $t_{1} t^{-1} \in P_{K}^{-}, m_{1} t_{1} t^{-1} m^{-1} \in$ $m_{1} \theta_{K}\left(t_{1} t^{-1}\right) m^{-1} U_{K}^{-}$. Since $U_{K}^{-} \subseteq U_{I \cap K}^{-}$,

$$
\begin{aligned}
\theta_{I \cap K}\left(m_{1} t_{1} t^{-1} m^{-1}\right) & =\theta_{I \cap K}\left(m_{1} \theta_{K}\left(t_{1} t^{-1}\right) m^{-1}\right) \\
& =\theta_{I \cap K}\left(m_{1} \theta_{K}\left(s_{1}^{-1} s\right) m^{-1}\right) \\
& =\theta_{I \cap K}\left(l_{1}^{-1} x_{1}^{-1} x l\right) .
\end{aligned}
$$

It follows that the multiplication on $\mathscr{M}$ is well defined. We next prove associativity. Let $a=x e_{I} y \in J_{I}, b=s e_{K} t \in J_{K}, c=u e_{N} v \in J_{N}, H=I \cap K \cap N$. Suppose $(a b) c \neq 0$. Then $y s \in U_{I}^{-} l_{1} l_{2} U_{K}$ for some $l_{1} \in L_{I}, l_{2} \in L_{K}$ and $a b=x l_{1} e_{I \cap K} l_{2} t$. Since $(a b) c \neq 0$,

$l_{2} t u \in U_{I \cap K}^{-} l_{3} l_{4} U_{N}$ for some $l_{3} \in L_{I \cap K}, l_{4} \in L_{N}$.

So $(a b) c=x l_{1} l_{3} e_{H} l_{4} v$. Now $U_{I \cap K}^{-}=U_{K}^{-}\left(U_{I}^{-} \cap L_{K}\right)$. So

$$
\begin{aligned}
t u & \in l_{2}^{-1} U_{I \cap K}^{-} l_{3} l_{4} U_{N}=l_{2}^{-1} U_{K}^{-}\left(U_{I}^{-} \cap L_{K}\right) l_{3} l_{4} U_{N} \\
& =U_{K}^{-} l_{2}^{-1}\left(U_{I}^{-} \cap L_{K}\right) l_{3} l_{4} U_{N} .
\end{aligned}
$$

So for some $z \in U_{I}^{-} \cap L_{K}$,

$$
t u \in U_{K}^{-} l_{2}^{-1} z l_{3} l_{4} U_{N} \text { and } b c=s l_{2}^{-1} z l_{3} e_{K \cap N} l_{4} v \text {. }
$$

Now

$$
\begin{aligned}
y s l_{2}^{-1} z l_{3} & \in U_{I}^{-} l_{1} l_{2} U_{K} l_{2}^{-1} z l_{3}=U_{I}^{-} l_{1} U_{K} z l_{3} \\
& =U_{I}^{-} l_{1} z l_{3} U_{K}, \quad \text { since } z l_{3} \in L_{K} \\
& =U_{I}^{-} l_{1} z l_{1}^{-1} l_{1} l_{3} U_{K} \\
& =U_{I}^{-} l_{1} l_{3} U_{K}, \text { since } z \in U_{I}^{-}, l_{1} \in L_{I} .
\end{aligned}
$$

Since $U_{K} \subseteq U_{K \cap N}$ and $l_{1} l_{3} \in L_{I}$, we see that

$$
a(b c)=x l_{1} l_{3} e_{H} l_{4} v=(a b) c .
$$

Similarly $(a b) c \neq 0$ implies that $a(b c)=(a b) c$. This shows that $\mathscr{M}$ is a monoid with group of units $G=J_{\Gamma}$. We proceed to show that $\mathscr{M}$ satisfies the two conditions for being a monoid of Lie type.

Let $x \in P\left(e_{I}\right)$. Then $x e_{I}=e_{I} x e_{I}$. So $e_{I} x e_{I} \neq 0, x \in P_{I}^{-} P_{I}$. Thus $x e_{I}=e_{I} x e_{I}=\theta_{I}(x) e_{I}$. Hence $x^{-1} \theta_{I}(x) \in P_{I}$. So $x \in P_{I}$. Clearly $L_{I} \subseteq$ $P\left(e_{I}\right)$. If $u \in U_{I}$, then $\theta_{I}(u)=1$. So $u e_{I}=e_{I}$ and $U_{I} \subseteq P\left(e_{I}\right)$. Hence $P_{I}=L_{I} U_{I} \subseteq P\left(e_{I}\right)$. So $P\left(e_{I}\right)=P_{I}$. Similarly $P^{-}\left(e_{I}\right)=P_{I}^{-}$and $e_{I} U_{I}^{-}=\left\{e_{I}\right\}$. 
It suffices now to show that all the idempotents of $J_{I}$ are conjugate. So let $f=x e_{I} y \in E(J)$. Then $e_{I} y x e_{I}=e_{I}$. So $y x \in P_{I}^{-} P_{I}, \theta_{I}(y x)=1$. Hence $y x \in U_{I}^{-} U_{I}$ and $y x=u v$ for some $u \in U_{I}^{-}, v \in U_{I}$. Thus $u^{-1} y=v x^{-1}$ and

$$
f=x e_{I} y=x v^{-1} e_{I} u^{-1} y=\left(x v^{-1}\right) e_{I}\left(v x^{-1}\right) .
$$

It follows that $e_{I}$ and $f$ are conjugate, proving the theorem.

Let $I \subseteq \Gamma$. Then $J_{I}^{0}=J_{I} \cup\{0\}$ is a subsemigroup of $\mathscr{M}$, which is completely 0 -simple. This means (since $\mathscr{M}$ is finite and regular) that $J_{I}^{0}$ has no proper ideals, cf. [4]. The maximal subgroup of $e_{I}$ is just $e_{I} L_{I} \cong L_{I}$ and $e_{I} \mathscr{M} e_{I} \cong$ $\mathscr{M}\left(L_{I}\right)$. The Rees theorem [4, Theorem 3.5] gives the structure of $J_{I}^{0}$ as a matrix semigroup over $L_{I}$. The multiplication is twisted by a sandwich matrix which we can describe easily. Let

$$
G / P_{I}^{-}=\left\{P_{I}^{-} a_{1}, \ldots, P_{I}^{-} a_{t}\right\}, \quad G / P_{I}=\left\{b_{1} P_{I}, \ldots, b_{t} P_{I}\right\} .
$$

The sandwich matrix is the $t \times t$ matrix $S=\left(\theta_{I}\left(a_{i} b_{j}\right)\right)$ where $\theta_{I}$ is taken to be zero on $G \backslash P_{I}^{-} P_{I} . J_{I}^{0}$ can be thought of as the set of all $t \times t$ matrices over $L_{I} \cup\{0\}$ with at most one nonzero entry and with the multiplication given by:

$$
A \circ B=A S B
$$

We will let $\mathscr{M}_{I}=G \cup J_{I} \cup\{0\}$. Then $\mathscr{M}_{I}$ is a submonoid of $\mathscr{M}$ and is itself a monoid of Lie type on $G$. We also note that $\mathscr{U}(\mathscr{M})=\left\{J_{I} \mid I \subseteq \Gamma\right\} \cup\{0\}$ and for all $I, K \subseteq \Gamma, J_{I}^{0} J_{K}^{0}=J_{I \cap K}^{0}$.

Let $M$ be any monoid of Lie type on $G, J \in \mathscr{U}(M), J \neq 0$. Let $J^{0}=$ $J \cup\{0\}$ with

$$
a \circ b= \begin{cases}a b & \text { if } a b \in J, \\ 0 & \text { if } a b \notin J .\end{cases}
$$

Then $J^{0}$ becomes a completely 0-simple semigroup. $M(J)=G \cup J^{0}$ (with obvious multiplication) is a monoid of Lie type. We note that in general $M(J)$ is not a submonoid of $M$, but is a submonoid of the Rees quotient monoid $M / \Omega$, where $\Omega=\{a \in M \mid J \nsubseteq M a M\}$. For instance, if $M=\mathscr{M}_{n}(F)$, then $J$ consists of all matrices of a particular rank. If $x \in G$, then we see by [11] that $e_{J} x e_{J} \in J$ if and only if $x \in P_{J}^{-} P_{J}$. We therefore have the following.

Corollary 1.2. Let $M$ be a monoid of Lie type, $J \in \mathscr{U}(M), J \neq 0, \lambda(J)=I$. Then there is a unique surjective homomorphism $\varphi: \mathscr{M}_{I} \rightarrow M(J)$ such that $\varphi\left(e_{I}\right)=e_{J}$ and $\varphi$ is the identity map on $G$.

\section{MODULAR REPRESENTATIONS}

Let $p$ denote the natural characteristic of $G, \kappa=\overline{\mathbb{F}}_{p}$. By a (modular) representations of $G$, we will mean in this section, a representation $\varphi: G \rightarrow$ $G L(n, \kappa)$. We will let $\kappa[G]$ denote the group algebra of $G$ over $\kappa$. By a $G$-module we will mean a $\kappa[G]$-module.

Let $M$ be a monoid of Lie type on $G$. We will mean by a (modular) representation of $M$, a homomorphism $\varphi: M \rightarrow \mathscr{M}_{n}(\kappa)$ such that $\varphi(1)=1$ and $\varphi(0)=0$. We will let $\kappa_{0}[M]$ denote the contracted semigroup algebra of $M$ over $\kappa$ (i.e., the zero of $M$ is the zero of $\left.\kappa_{0}[M]\right)$. Then the representations of $M$ are in a natural 1-1 correspondence with the nonzero $\kappa_{0}[M]$-modules. By an $M$-module we will mean a $\kappa_{0}[M]$-module. We will use the same notation for any semigroup with zero. 
Proposition 2.1. Let $G$ be a reductive group defined over $\kappa$ and $G_{\sigma}$ an associated finite group of Lie type. Let $\psi: G \rightarrow G L(n, \kappa)$ be a representation, $\varphi$ the restriction of $\psi$ to $G_{\sigma}$. Let $B, B^{-}$be opposite $\sigma$-stable Borel subgroups of $G, T=B \cap B^{-}$. Then there is a minimal nonzero idempotent $e_{\varphi} \in E(\overline{\kappa \psi(T)})$ such that $\psi(B) \subseteq P\left(e_{\varphi}\right), \psi\left(B^{-}\right) \subseteq P^{-}\left(e_{\varphi}\right)$. For $g \in G, a \in \mathscr{M}_{n}(\kappa)$, let $g a=\psi(g) a, a g=a \psi(g)$. Then

$$
M_{\varphi}=G_{\sigma} \cup G_{\sigma} e_{\varphi} G_{\sigma} \cup\{0\}
$$

is a monoid of Lie type on $G_{\sigma}$ and there is a representation $\hat{\varphi}: M_{\varphi} \rightarrow \mathscr{M}_{n}(\kappa)$ with $\hat{\varphi}(g)=\varphi(g)$ for $g \in G_{\sigma}, \hat{\varphi}\left(e_{\varphi}\right)=e_{\varphi}$.

Proof. Let $G_{1}=\kappa^{*} \psi(G)$. Then $G_{1}$ is a reductive group. Let $M_{1}=\bar{G}_{1}$, the closure in $\mathscr{M}_{n}(\kappa)$. Then $M_{1}$ is a regular linear algebraic monoid and the existence of $e=e_{\varphi}$ follows from [10, Chapter 9]. Moreover, if $T_{1}=\kappa^{*} \psi(T)$, then $e \psi(G) e=e T_{1} \cup\{0\}$. In particular, $e U^{-}=\{e\}=U e$ where $U, U^{-}$ denote the unipotent radicals of $B, B^{-}$, respectively. Let $N=N_{G}(T)$. Then for all $n \in N$, either $e n=n e$ or else ene $=\left(e \cdot n e n^{-1}\right) n=0$. Let $P_{1}=$ $\left\{x \in G_{\sigma} \mid x e=e x e\right\}, P_{1}^{-}=\left\{x \in G_{\sigma} \mid e x=e x e\right\}$. Then $P_{1}, P_{1}^{-}$are parabolic subgroups of $G_{\sigma}$ containing $B_{\sigma}$ and $B_{\sigma}^{-}$respectively. Moreover, for $n \in$ $N_{\sigma}, n \in P_{1}$ if and only if $n \in P_{1}^{-}$. It follows that $P_{1}$ and $P_{1}^{-}$are opposite parabolic subgroups of $G_{\sigma}$. Let $L_{1}=P_{1} \cap P_{1}^{-}$. Then $P_{1}=L_{1} U_{1}, P_{1}^{-}=$ $L_{1} U_{1}^{-}, U_{1} \subseteq U, U_{1}^{-} \subseteq U^{-}$. So $U_{1} e=\{e\}=e U_{1}^{-}$. Let $x \in G_{\sigma}$. Suppose $x \notin P_{1}^{-} P_{1}$. Then by the Bruhat decomposition, $x=a n b$ for some $a \in P_{1}^{-}, n \in$ $N_{\sigma}, b \in P_{1}, n \notin L_{1}$. So $e n e=0$ and

$$
\text { exe }=(e a e)(e n e)(e b e)=0 \text {. }
$$

If $x \in P_{1}^{-} P_{1}=U_{1}^{-} L_{1} U_{1}$ then for some $l \in L_{1}, u \in U_{1}^{-}, v \in U_{1}, x=u l v$ and

$$
\text { exe }=\text { eulve }=e l e=e l=l e .
$$

It follows that $M_{\varphi}$ is a monoid. It suffices to show that all the idempotents of $G_{\sigma} e G_{\sigma}$ are conjugate in $M_{\varphi}$. So let $x, y \in G_{\sigma}$ such that $f=x e y \in E\left(M_{\varphi}\right)$. Then eyxe $=e$. So $y x \in P_{1}^{-} P_{1}$. Hence $y x=u l v, u \in U_{1}^{-}, v \in U_{1}, l \in L_{1}$. So ele $=e y x e=e, u^{-1} y=l v x^{-1}$ and

$$
f=x e y=x v^{-1} l^{-1} e u^{-1} y=\left(x v^{-1} l^{-1}\right) e\left(l v x^{-1}\right)
$$

Hence $e$ and $f$ are conjugate in $M_{\varphi}$. Thus $M_{\varphi}$ is a monoid of Lie type and the proof is complete.

Let $G$ be a (finite) group of Lie type with Coxeter graph $\Gamma$. By [5, Theorems 5.7, 6.15] the irreducible modular representations of $G$ are in 1-1 correspondence with ordered pairs $(I, \chi)$ where $I \subseteq \Gamma$ and $\chi: L_{I} \rightarrow \kappa^{*}$ any homomorphism. If $V$ is an irreducible $\kappa[G]$-module, then there is a unique one-dimensional subspace $Y$ of $V$ which is stabilized by $B . I \subseteq \Gamma$ is such that $P_{I}=\{x \in G \mid x Y=Y\}$. Then $Y$ is a one-dimensional $\kappa\left[L_{I}\right]$ module, yielding $\chi: L_{I} \rightarrow \kappa^{*}$.

By Theorem 1.1, the non-zero $\mathscr{J}$-classes of $\mathscr{M}=\mathscr{M}(G)$ are $J_{I}(I \subseteq \Gamma)$ and the maximal subgroup of $e_{I}$ is $e_{I} L_{I} \cong L_{I}$. Hence by [4, Theorems 5.33, 5.51] the irreducible modular representations of $\mathscr{M}$ are in 1-1 correspondence with ordered pairs $(I, \varphi)$ where $I \subseteq \Gamma$ and $\varphi$ is a modular irreducible representation of $L_{I}$. Let $V$ be an irreducible $\kappa_{0}[\mathscr{M}]$-module. Then there is a minimum $I \subseteq \Gamma$ such that $J_{I} V \neq 0$, and $e_{I} V$ is an irreducible $L_{I}$-module yielding $\varphi$. 
Theorem 2.2. Every irreducible modular representation of $\mathscr{M}=\mathscr{M}(G)$ restricts to an irreducible representation of $G$. An irreducible representation of $G$ of type $(I, \chi)$ extends to $2^{|\Gamma \backslash I|}$ inequivalent irreducible representations of $\mathscr{M}$. If $\alpha_{I}$ denotes the number of homomorphisms from $L_{I}$ to $\kappa^{*}$, then the number of inequivalent irreducible representations of $\mathscr{M}$ is $\sum_{I \subseteq \Gamma} 2^{|\Gamma \backslash I|} \alpha_{I}$.

Proof. Let $I \subseteq \Gamma, \chi: L_{I} \rightarrow \kappa^{*}$ a homomorphism. We then have an associated irreducible $G$-module $V$. Let $\varphi$ be the corresponding representation of $G$. By [20], $\varphi$ is the restriction of a representation of the associated reductive group. By Proposition 2.1, we can construct the monoid of Lie type $M_{\varphi}$. Then $V$ is an $M_{\varphi}$-module. By Corollary $1.2, V$ then becomes an irreducible $\mathscr{M}_{I}$-module such that $B$ stabilizes the one-dimensional subspace $e_{I} V$ and $P_{I}$ is the stabilizer of $e_{I} V$. Since $J_{I}^{0}$ is an ideal of $\mathscr{M}_{I}, V$ is also an irreducible $J_{I}^{0}$-module. Let $\Omega=$ $\cup\left\{J_{K} \mid I \nsubseteq K\right\} \cup\{0\}$. Then $\Omega$ is an ideal of $\mathscr{M}$ and $J_{I}^{0}$ is an ideal of the Rees quotient semigroup $\mathscr{M} / \Omega$. Thus $V$ becomes an $\mathscr{M} / \Omega$-module and hence also an $\mathscr{M}$-module. Now $J_{I} V \neq 0, J_{K} V=0$ for $I \nsubseteq K$. Now let $I \subseteq K$. Then since $e_{K} \geq e_{I}, J_{K} V \neq 0$. Since $\mathscr{M}_{K}$ is a submonoid of $\mathscr{M}$, containing $G, V$ is also an irreducible $\mathscr{M}_{K}$-module. Hence as above, $V$ becomes an irreducible $\mathscr{M}$-module in a new way such that $J_{K} V \neq 0, J_{K^{\prime}} V=0$ for $K \nsubseteq K^{\prime}$. We have thus produced $2^{|\Gamma \backslash I|}$ inequivalent irreducible $\mathscr{M}$-modules which are all the same as $G$-modules. Hence we have produced

$$
\sum_{I \subseteq \Gamma} 2^{|\Gamma \backslash I|} \alpha_{I}
$$

inequivalent irreducible representations of $\mathscr{M}$, each of which restricts to an irreducible representation of $G$. The number of inequivalent irreducible representations of $L_{I}$ is $\sum_{K \subseteq I} \alpha_{K}$. The number of irreducible representations of $\mathscr{M}$ is therefore

$$
\sum_{I \subseteq \Gamma} \sum_{K \subseteq I} \alpha_{K} .
$$

Since the numbers (1), (2) are equal, the proof is complete.

Example 2.3. Let $G=S L\left(n, \mathbb{F}_{q}\right)$. The number of irreducible modular representations of $G$ is equal to $q^{n-1}$, cf. [20]. The number of irreducible modular representations of $\mathscr{M}$ is equal to $\sum_{i=0}^{n-1} 2^{i} q^{n-1-i}=\frac{q^{n}-2^{n}}{q-2}$.

We now use a result of Clifford [2,3] to obtain some information about the degree of an irreducible modular representation of $G$.

Corollary 2.4. Let $\varphi$ be an irreducible modular representation of $G$ of type $(I, \chi)$ where $I \subseteq \Gamma, \chi: L_{I} \rightarrow \kappa^{*}$ a homomorphism. Let

$$
G / P_{I}^{-}=\left\{P^{-} a_{1}, \ldots, P^{-} a_{t}\right\}, \quad G / P_{I}=\left\{b_{1} P, \ldots, b_{t} P\right\} .
$$

If $a_{i} b_{j} \in P_{I}^{-} P_{I}$, let $\alpha_{i j}=\chi\left(\theta_{I}\left(a_{i} b_{j}\right)\right)$. If $a_{i} b_{j} \notin P_{I}^{-} P_{I}$, let $\alpha_{i j}=0$. Then the degree of $\varphi$ is equal to the rank of $\left(\alpha_{i j}\right)$.

Proof. Let $V$ be the corresponding $G$-module. Then by Theorem 2.2, $V$ is also a $J_{I}^{0}$-module. By Clifford $[2,3]$ or $[4$, Theorem 5.46], $\operatorname{dim} V$ is equal to the rank of $\chi(S)$, where $S$ is the sandwich matrix of $J_{I}^{0}$. We have already seen that $S=\left(\theta_{I}\left(a_{i} b_{j}\right)\right)$ where $\theta_{I}$ is taken to be zero on $G \backslash P_{I}^{-} P_{I}$. The result follows. 
Remark 2.5. Actually by [4, Chapter 5] we see that $\varphi$ can be obtained from the full rank factorization of the $t \times t$ matrix $\left(\alpha_{i j}\right)$.

Corollary 2.6. The modular Steinberg representation of $G$ extends to an idempotent separating representation $\varphi$ of $\mathscr{M}$. The representation $\varphi$ can also be obtained (via Clifford [2]) from the trivial representation of $T=L_{\varnothing}$.

Proof. Since the Steinberg representation is of type $(\varnothing, 1)$, we see by Theorem 2.2 that it has an extension to a representation $\varphi$ of $\mathscr{M}$ such that $\varphi\left(e_{\varnothing}\right) \neq 0$ and the corresponding $L_{\varnothing}$-module is the trivial module. Hence it suffices to show that $\varphi$ is idempotent separating. Suppose $\varphi(e)=\varphi(f)$ for some idempotents $e \neq f$. Then either $e \mathscr{M} \neq f \mathscr{M}$ or $\mathscr{M} e \neq \mathscr{M} f$. By symmetry assume $e \mathscr{M} \neq f \mathscr{M}$. By [11, Corollary 2.17] there exist $e_{1}, f_{1} \in E(\mathscr{M})$ such that $e \mathscr{M}=e_{1} \mathscr{M}, f \mathscr{M}=f_{1} \mathscr{M}$ and $e_{1} f_{1}=f_{1} e_{1}$. Let $h=e_{1} f_{1}$. Then either $e_{1} \neq h$ or $f_{1} \neq h$. By symmetry let $e_{1} \neq h$. Then $e_{1}>h$ and $\varphi\left(e_{1}\right)=\varphi(h)$. So $h \neq 0$. By [11, Corollary 2.14] we can assume that $e_{1}=e_{I}, h=e_{K}, K \subset I$. There exists $\sigma=n T \in I \backslash K$. So

$$
0=\varphi\left(e_{K} n e_{K}\right)=\varphi\left(e_{I} n e_{I}\right)=\varphi\left(e_{I} n\right) \neq 0 .
$$

This contradiction completes the proof.

Corollary 2.7. Let $M$ be any monoid of Lie type on $G$. Then any irreducible modular representation of $M$ restricts to an irreducible representation of $G$.

Proof. Let $V$ be an irreducible $M$-module. Then there is a minimum $J \in$ $\mathscr{U}(M)$ such that $J V \neq 0$. Let $\Omega=\{a \in M \mid J \nsubseteq M a M\}$. Then $\Omega$ is an ideal of $M$ and $V$ is an irreducible $M / \Omega$-module. Since $J^{0}$ is an ideal of $M / \Omega, V$ is an irreducible $J^{0}$-module. Since $J^{0}$ is an ideal of $M(J), \quad V$ is also an irreducible $M(J)$-module. By Corollary $1.2, V$ is an irreducible $\mathscr{M}_{I}$-module where $I=\lambda(J)$. Hence $V$ is an irreducible $\mathscr{M}$-module. By Theorem 2.2, $V$ is an irreducible $G$-module. This completes the proof.

Irreducible modular representations of $\mathscr{M}_{n}\left(\mathbb{F}_{p}\right)$ were determined by Harris and Kuhn [7]. By Corollary 1.2 and Theorem 2.2, we have

Corollary 2.8. Let $M$ be a monoid of Lie type on $G$ and $\varphi: G \rightarrow G L(n, \kappa)$ an irreducible modular representation of type $(I, \chi)$. Then the inequivalent irreducible representations of $M$, extending $\varphi$, are in 1-1 correspondence with $0 \neq J \in \mathscr{U}$ such that $\lambda(J)=K \supseteq I$ and so that the following diagram can be completed:

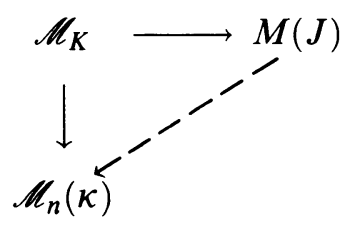

Remark 2.9. By Corollary 2.8, it follows that the irreducible modular representations of $M$ can be enumerated by analyzing the combinatorial system $(\mathscr{U}, \lambda)$ and the irreducible modular representations of $G$. In particular the results of Harris and Kuhn [7, §6] can be recovered.

Remark 2.10. The referee points out that Corollary 2.7 is not valid for indecomposable representations. A counterexample is provided by $M=\mathscr{M}_{2}\left(\mathbb{F}_{2}\right), G=$ 
$G L\left(2, \mathbb{F}_{2}\right), V=\left\langle x^{2}, y^{2}, x y\right\rangle \subseteq \kappa[x, y]$. The referee further informs us that Leonid Krop has studied such examples in detail.

\section{COMPLEX REPRESENTATIONS}

Let $G$ be a finite group of Lie type. By a representation of $G$, we will mean in this section, a homomorphism $\varphi: G \rightarrow G L(n, \mathbb{C})$. If $M$ is a finite monoid of Lie type on $G$, we will mean by a representation of $M$, a homomorphism $\varphi: M \rightarrow \mathscr{M}_{n}(\mathbb{C})$ such that $\varphi(1)=1$ and $\varphi(0)=0$.

An irreducible representation $\varphi: G \rightarrow G L(n, \mathbb{C})$ is cuspidal if for any $I \subsetneq \Gamma$,

$$
\sum_{u \in U_{I}} \varphi(u)=0 \text {. }
$$

Then Harish-Chandra observed, cf. [1, Chapter 9] that for any irreducible representation $\varphi$ of $G$, there is a subset $I \subseteq \Gamma$ and an irreducible cuspidal representation $\theta$ of $L_{I}$ such that if $\bar{\theta}$ is the lift of $\theta$ from $L_{I}$ to $P_{I}$ (i.e., $\bar{\theta}$ is trivial on $U_{I}$ ), then $\varphi$ is a component of the induced representation $\bar{\theta}_{P_{I}}^{G}$. In this way the irreducible representations of $G$ are classified according to which Levi subgroup $L_{I}$ they come from. This philosophy of Harish-Chandra along with a submonoid of $\mathscr{M}(G)$ was used by Okniński and one of the authors [8] to show that any complex representation of a monoid of Lie type is completely reducible. This result along with the decomposition of [16] are used by Solomon [18] to begin a program of constructing Hecke algebras for monoids of Lie type.

Let $\mathscr{M}=\mathscr{M}(G)$. Then by Theorem 1.1 the nonzero $\mathscr{J}$-classes of $\mathscr{M}$ are $J_{I}(I \subseteq \Gamma)$. Moreover, the maximal subgroup of $e_{I}$ is $e_{I} L_{I} \cong L_{I}$. Hence by [4, Theorems 5.33, 5.51], the irreducible representations $\varphi$ of $\mathscr{M}$ are in a natural 1-1 correspondence with $(I, \theta)$ where $I \subseteq \Gamma$ and $\theta$ is an irreducible representation of $L_{I}$. We will say that $\varphi$ is cuspidal if $\theta$ is cuspidal. By [8, Corollary 2.7] $\varphi$ restricted to $G$ is just the induced representation $\bar{\theta}_{P_{I}}^{G}$ where $\bar{\theta}$ is the lift of $\theta$ to $P_{I}$. We therefore have the following interpretation of Harish-Chandra's philosophy:

Theorem 3.1. Any complex irreducible representation of $G$ is a component of an irreducible cuspidal representation of $\mathscr{M}$, restricted to $G$.

Remark 3.2. Unlike in the situation of modular representations, the above theorem only gives us induced representations and not irreducible representations of $G$. For example, unlike Corollary 2.6, it does not give us the Steinberg representation. For this reason it may be worthwhile to look for natural monoids having $G$ as the group of units, whose complex semigroup algebras are not semisimple!

Conjecture 3.3. Theorem 2.1 is valid for representation over fields of characteristic not equal to the natural characteristic of $G$.

\section{REDUCTIVE MONOIDS AND FINITE MONOIDS}

Let $M$ be a reductive, algebraic monoid with unit group $G=G(M)$. By this we mean that $M=\bar{G}$ is a linear algebraic monoid, cf. [10], with $G$ being a reductive group. If the center $Z G$ of $G$ is one dimensional, we say that $M$ is semisimple. We assume throughout that the characteristic of the underlying 
ground field is $p>0$. Let $\sigma$ be an endomorphism of $M$. Let $G_{\sigma}=\{g \in$ $G \mid \sigma(g)=g\}$ and $M_{\sigma}=\{x \in M \mid \sigma(x)=x\}$. Define $1-\sigma: G \rightarrow G$ by $(1-\sigma)(g)=g \sigma(g)^{-1}$. For $X \subseteq M$, let $X_{\sigma}=\{x \in X \mid \sigma(x)=x\}$. If $G=T$ is a torus we say that $M=\bar{T}$ is a $D$-monoid. We denote by $X(T)$ and $X(\bar{T})$ the character group and monoid of $T$ and $\bar{T}$, respectively.

Proposition 4.1. The following are equivalent:

(a) $\sigma$ is a finite morphism and $G_{\sigma}$ is a finite group.

(b) $(1-\sigma)(G)=G$ and $\sigma(Z)=Z$ for some $Z=\bar{T} \subseteq M$ a maximal D-submonoid.

Proof. Assume (a). If $G_{\sigma}$ is finite then by [20] there exists $T \subseteq G$ a maximal torus such that $\sigma(T)=T$. If $\sigma$ is finite then $\sigma(\bar{T})=\bar{T}$ since finite morphisms are closed. Furthermore, by Lang's theorem [20], $(1-\sigma)(G)=G$. Assume (b). If $(1-\sigma)(G)=G$ then $G_{\sigma}$ is finite, and by [19] there exists $T \subseteq G$ a maximal torus such that $\sigma(T)=T$. By assumption, $\sigma(\bar{T})=\bar{T}$ since all maximal tori are conjugate. Thus, $\sigma(E(\bar{T}))=E(\bar{T})$. Hence, $\sigma$ is injective on $E(\bar{T})$ and so $\sigma \mid \bar{T}$ is finite. Thus, by $[15,2.1$ and 6.3] $\sigma$ is finite.

Corollary 4.2. Assuming $G_{\sigma}$ finite, the following are equivalent:

(a) $\sigma: M \rightarrow M$ is finite.

(b) If $\sigma(T)=T$ then in $\sigma^{*}: X(\bar{T}) \rightarrow X(\bar{T}), \sigma^{*}(X(\bar{T})) \subseteq X(\bar{T})$ satisfies $n X(\bar{T}) \subseteq \sigma^{*}(X(\bar{T}))$ for some $n>0$.

Proof. By [10, Theorem 8.14], $\sigma \mid \bar{T}$ is finite if and only if $n X(\bar{T}) \subseteq \sigma^{*}(X(\bar{T}))$ for some $n>0$.

Assume that $\sigma$ is a finite morphism with $G_{\sigma}$ finite. We now investigate the important structural properties of $M_{\sigma}$. We let $\Lambda=\Lambda(M)$ denote a cross section lattice of $M,[10$, Chapter 9]. Thus $\Lambda$ consists of order-preserving idempotent representatives of $\mathscr{J}$-classes $(=G \times G$ orbits) of $M$. The Green's relation $\mathscr{R}$ on $M$ is defined as: $a \mathscr{R} b$ if and only if $a M=b M$.

Theorem 4.3. (a) $M_{\sigma}$ is finite.

(b) $M_{\sigma}$ is unit regular, i.e. $M_{\sigma}=E\left(M_{\sigma}\right) G_{\sigma}$.

(c) If $T \subseteq B$ satisfies $\sigma(T)=T$ and $\sigma(B)=B$ then $M_{\sigma}=\bigcup_{e \in \Lambda_{\sigma}} G_{\sigma} e G_{\sigma}$ and $E_{\sigma}=E\left(M_{\sigma}\right)=\bigcup_{g \in G_{\sigma}} g \Lambda_{\sigma} g^{-1}$ where $\Lambda_{\sigma}=\{e \in E(\bar{T}) \mid \sigma(e)=$ $e, B e=e B e\}$.

(d) If $e \in E_{\sigma}$ then $C_{G_{\sigma}}^{r}(e)$ and $C_{G_{\sigma}}^{l}(e)$ are opposite parabolic subgroups of $G_{\sigma}$.

(e) If $e, f \in E_{\sigma}$ and $e M_{\sigma}=f M_{\sigma}$ or $M_{\sigma} e=M_{\sigma} f$ then there exists $g \in G_{\sigma}$ such that geg $^{-1}=f$.

(f) If $e \in \Lambda_{\sigma}$ then $U e=e U^{-}=\{e\}$, where $U \triangleleft C_{G_{\sigma}}^{r}(e)$ and $U^{-} \triangleleft C_{G_{\sigma}}^{l}(e)$ are the unipotent radicals.

Proof. We first prove (b). Let $x \in M_{\sigma}$, so $\sigma(x)=x$. Thus, $\sigma(R)=R$ where $R=x G$ is the $\mathscr{R}$-class of $x$. If $x=e x, e \in E(R)$, then $\sigma\left(C_{G}^{r}(e)\right)=C_{G}^{r}(e)$. But then $C_{G}^{r}(e) \times E(R) \rightarrow E(R)$ is transitive, while $C_{G}(f)$ is connected for all $f \in E(R)$ [10, Corollary 6.18]. Thus, by [19, I, 2.7(b)], $C_{G_{\sigma}}^{r}(e) \times E(R)_{\sigma} \rightarrow$ $E(R)_{\sigma}$ is transitive, and $E(R)_{\sigma} \neq \varnothing$. Hence, $x=e y$ for some $e \in E(R)_{\sigma}, y \in$ $G$. Now let $X=\{g \in G \mid e g=x\}, H=\{h \in G \mid e h=e\}$. Then $H \times H \rightarrow X$ 
is transitive and $\sigma$ acts accordingly. Thus, by $[19, \mathrm{I}, 2.7(\mathrm{a})], X_{\sigma} \neq \varnothing$. This shows that we can choose $g \in G_{\sigma}$ and so $M_{\sigma}$ is unit regular.

Proof of (a). Let $f \in E_{\sigma}$, and let $Z=C l_{G}(f)=\left\{g f g^{-1} \mid g \in G\right\}$. Then $\sigma(Z)=Z$. But then $G \times Z \rightarrow Z$ is transitive and $\sigma$ acts as in [19]. Thus by $[19, \mathrm{I}, 2.7(\mathrm{~b})] G_{\sigma} \times Z_{\sigma} \rightarrow Z_{\sigma}$ is transitive, and so $Z_{\sigma}$ is finite. But the number of such $Z$ 's is finite. Hence, $E_{\sigma}$ is finite. Finally, $M_{\sigma}=G_{\sigma} E_{\sigma} G_{\sigma}$ is finite.

Proof of (c). We have $M_{\sigma}=G_{\sigma} E_{\sigma} G_{\sigma}$. If $\sigma(e)=e$ then $\sigma(L)=L$ where $L=$ $C_{G}(e)$. Thus, there exists $T \subseteq B_{0} \subseteq L$, a maximal torus and Borel subgroup respectively, such that $\sigma(T)=T$ and $\sigma\left(B_{0}\right)=B_{0}$. But also $\sigma\left(R_{u}\left(C_{G}^{r}(e)\right)\right)=$ $R_{u}\left(C_{G}^{r}(e)\right)$. Now $B=B_{0} R_{u}\left(C_{G}^{r}(e)\right)$ is a Borel subgroup of $G$, and $\sigma(B)=B$. Hence we have found a pair $(T, B)$ such that $\sigma(T) \subseteq T, \sigma(B) \subseteq B, T \subseteq B$ and $T \subseteq C_{G}(e)$. Hence $\Lambda=\left\{f \in E(\bar{T}) \mid B \subseteq C_{G}^{r}(f)\right\}$ is a cross section lattice of $M$ such that $\sigma(\Lambda) \subseteq \Lambda$ and $e \in \Lambda_{\sigma}$. From the proof of (a) we know that if $e, f \in M_{\sigma}$ are conjugate in $M$ then they are conjugate in $M_{\sigma}$. So $E_{\sigma}=\bigcup_{g \in G_{\sigma}} g \Lambda_{\sigma} g^{-1}$. Hence, $M_{\sigma}=\bigcup_{e \in \Lambda_{\sigma}} G_{\sigma} e G_{\sigma}$. The union is disjoint since $\Lambda_{\sigma} \subseteq \Lambda$ and $G_{\sigma} \subseteq G$.

Proof of (d). This follows from [10, Theorem 7.1] and the observations (i) if $P \subseteq G$ is parabolic and $\sigma(P)=P$, then $P_{\sigma} \subseteq G_{\sigma}$ is parabolic, (ii) if $P$ and $P^{-}$are opposite in $G$ and $\sigma(P)=P, \sigma\left(P^{-}\right)=P^{-}$then $P_{\sigma}$ and $P_{\sigma}^{-}$are opposite in $G_{\sigma}$.

$\operatorname{Proof}$ of (e). This is another application of [19]. Let $e, f \in E_{\sigma}, e \mathscr{R} f$ in $M_{\sigma}$. Then $e \mathscr{R} f$ in $M$. Thus $G \times Z \rightarrow Z$ is transitive, where $Z=C l_{G}(e)=$ $C l_{G}(f)$. Since $C_{G}(e)$ is connected, $G_{\sigma} \times Z_{\sigma} \rightarrow Z_{\sigma}$ is transitive.

Proof of (f). If $e \in \Lambda_{\sigma}$ and $P=C_{G}^{r}(e)$ then $U=R_{u}(P)_{\sigma}$. But $R_{u}(P) e=$ $\{e\}$.

Remark 4.4. Because of (b), (d), (e) and (f) $M_{\sigma}$ is, in the terminology of $\S 1$, a monoid of Lie type. In particular, there is a Bruhat type decomposition for $M_{\sigma}$ which follows formally from Theorem 4.3 above and the results of [11]. In detail, if $\sigma(T)=T, \sigma(B)=B, T \subseteq B$ and $\Lambda=\left\{e \in E(\bar{T}) \mid B \subseteq C_{G}^{r}(e)\right\}$, then by $[11, \S 4], M_{\sigma}=\bigcup_{r \in \mathfrak{R}^{\prime}} B_{\sigma} r B_{\sigma}$, where $\mathfrak{R}^{\prime}=\left\langle W_{\sigma}, E(\bar{T})_{\sigma}\right\rangle$. But the union is disjoint since $\mathfrak{R}^{\prime} \subseteq \mathfrak{R}=\langle W, E(\bar{T})\rangle$ and $B_{\sigma} \subseteq B$. Also $\mathfrak{R}^{\prime} \subseteq \mathfrak{R}_{\sigma}$. Conversely, $\mathfrak{R}_{\sigma} \subseteq \mathfrak{R}^{\prime}$ since $x \in \mathfrak{R}_{\sigma}$ implies $\sigma: T \cdot x \rightarrow T \cdot x$ has a fixed point [19, I, 2.7(a)] say $x$, and so $x \in B_{\sigma} r B_{\sigma}$ for some $r \in \mathfrak{R}^{\prime}$. Thus, $x=r$ by the above disjointedness argument.

Hence we obtain

Corollary 4.5. $M_{\sigma}=\bigcup_{r \in \mathfrak{R}_{\sigma}} B_{\sigma} r B_{\sigma}$. Furthermore, $\mathfrak{R}_{\sigma}=\left\langle W_{\sigma}, E(\bar{T})_{\sigma}\right\rangle$, and the union is disjoint.

Remark 4.6. One can use Corollary 4.5 to obtain a formula for the order of $M_{\sigma}$ [17]. One could probably refine that formula significantly.

Our task now is to construct, for each finite group $G_{\sigma}$ of Lie type a monoid $M_{\sigma}$ (for some reductive monoid $M$ with $\sigma: M \rightarrow M$ ) with the following properties:

(1) $G\left(M_{\sigma}\right)$ is a central extension of $G_{\sigma}$.

(2) The map $e \mapsto C_{G_{\sigma}}(e)=P_{I(e)}$ induces an order preserving bijection from $\Lambda_{\sigma} \backslash\{0\}$ to $2^{\Gamma}$. Here $\Gamma$ is the set of simple involutions relative 
to $T_{\sigma}$ and $B_{\sigma}$, and $I(e) \subseteq \Gamma$ is such that $C_{G_{\sigma}}^{r}(e)=B_{\sigma} W_{I(e)} B_{\sigma}$. By the results of [13] one can obtain reductive algebraic monoids with these properties by choosing a high weight in general position. The resulting monoid is a cone on the canonical compactification. This compactification was introduced in [6] because of its superior intersection theoretic properties.

For most pairs $(G, \sigma)$ the construction of $M$ as above is straightforward, but for $(G, \sigma)$ of Ree or Suzuki type, one cannot use a semisimple monoid, because the requirement that $\sigma: M \rightarrow M$ be a finite morphism would imply that $\sigma^{2}(t)=t^{p^{2 a+1}}$ for all $t \in Z(G(M))^{0}$ and some $a>0$. This is not possible.

Suppose that $G$ is a simple algebraic group and $\sigma: G \rightarrow G$ is of Chevalley or Steinberg type. So if $\sigma(T)=T$ and $\sigma(B)=B$ then $\sigma^{*}(\alpha)=q \rho^{*}(\alpha)$ for all $\alpha \in \Delta$ where $\rho: G \rightarrow G$ is some outer automorphism ( $\rho=$ id is allowed) such that $\rho(T)=T$ and $\rho(B)=B$. Let $(X, \Phi)$ be the root system of $G \times k^{*}$ where $X=X(T) \oplus \mathbb{Z}$, and $\Phi \subseteq X(T)$ is the set of roots of $G$. Choose a dominant weight $\lambda=\sum_{i=1}^{r} a_{i} \lambda_{i}$ such that $\sigma^{*}(x)=q \lambda$ and $a_{i}>0$ for all $i$. Let $(\lambda, 1) \in X$ and extend $\sigma^{*}$ to $X$ via $\sigma_{1}^{*}(\mu, \gamma)=\left(\sigma^{*}(\mu), q \gamma\right)$. Then $\sigma_{1}^{*}$ is induced from $\sigma_{1}: G \times k^{*} \rightarrow G \times k^{*}, \sigma_{1}(g, t)=\left(\sigma(g), t^{q}\right)$. Let $C \subseteq X$ be the smallest polyhedral cone containing $\{(w(\lambda), 1 \mid w \in W\}$. Then $C$ is $W$-invariant and $\sigma_{1}^{*}(C)=q C$. By [15, Theorem 6.5] there exists a semisimple monoid $M$ with unit group $G \times k^{*}$ such that $X\left(\bar{T}_{1}\right)=C \subseteq X$, where $\bar{T}_{1} \subseteq M$ is the closure in $M$ of $T_{1}$. By [15, Corollary 4.5] and Corollary 1.2 above, $\sigma_{1}: G \times k^{*} \rightarrow G \times k^{*}$ extends to a finite morphism $\sigma_{1}: M \rightarrow M$ such that $\left(G \times k^{*}\right)_{\sigma}$ is a finite group. Changing notation, let $\sigma=\sigma_{1}$ and $G=G_{1}$.

Theorem 4.7. Let $\Gamma$ be the set of simple involutions of $W_{\sigma}$ relative to $B_{\sigma}$. Then $M_{\sigma}$ satisfies the following properties:

(a) $\Lambda_{\sigma} \backslash\{0\} \cong 2^{\Gamma}$, via the map $e \mapsto I(e), C_{G_{\sigma}}^{r}(e)=P_{I(e)}, I(e) \subseteq \Gamma$.

(b) If $I(e)=I, I(f)=K \subseteq \Gamma$ let $J_{I}=G_{\sigma} e G_{\sigma}$ and $J_{K}=G_{\sigma} f G_{\sigma}$. Then $J_{I} J_{K} \subseteq J_{I \cap K} \cup\{0\}$.

Proof. By $[1,1.18]$, the standard parabolics of $G_{\sigma}$ are $\left\{P_{\sigma} \mid P \supseteq B\right.$ and $\sigma(P)=$ $P\}$. Conversely, $\Lambda_{\sigma}=\{e \in \Lambda \mid \sigma(e)=e\}=\left\{e \in \Lambda \mid \sigma\left(C_{G}^{r}(e)\right)=C_{G}^{r}(e)\right\}$. Hence $\Lambda_{\sigma} \backslash\{0\} \rightarrow 2^{\Gamma}$ is bijective, and order preserving since by [13, Lemma 4.12], $\Lambda \backslash\{0\} \rightarrow\{P \mid P \supseteq B\}$ is order preserving. This proves (a).

For (b) it suffices to prove the corresponding statement for $M$, since for $e \in \Lambda_{\sigma} \backslash\{0\}, G e G \cap M_{\sigma}=G_{\sigma} e G_{\sigma}$. Let $e_{0} \in \Lambda \backslash\{0\}$ be the minimal element. Since $C_{W}\left(e_{0}\right)=\{1\}$, it follows that $\Lambda \backslash\{0\}=\left\{f \in E(\bar{T}) \mid f e_{0}=e_{0} f=e_{0}\right\}$. So let $x \in J_{I}, y \in J_{K}$. Then we can write $x=g e g^{\prime}, y=h^{\prime} f h$ where $e, f \in \Lambda \backslash\{0\}$ and $g, h, g^{\prime}, h^{\prime} \in G$. Thus, $x y=g e g^{\prime} h^{\prime} f h=g e h_{1} w h_{2} f h$, where $b_{1} \in B^{-}, b_{2} \in B$ and $w \in W$. But $e, f \in \Lambda$, so $x y=g b_{1}^{\prime} e w f b_{2}^{\prime} h$ for some $b_{1}^{\prime} \in B^{-}, b_{2}^{\prime} \in B$. If $e w f w^{-1}=0$ then $x y=0$. If $e w f w^{-1} \neq 0$ then $e, w f w^{-1} \in \Lambda^{\prime}$ for some cross section lattice $\Lambda^{\prime} \subseteq E(\bar{T})$. But $\Lambda^{\prime v}=\Lambda$ for some $v \in W$. Thus, $e^{v}, e, f^{v w}, f \in \Lambda$, and so $e^{v}=e$ and $f^{v w}=f$. Hence, $x y \in J_{I \cap K}$ because $\left(e f^{w}\right)^{v}=e f$.

We now turn to the task of constructing monoids as in Theorem 4.7 for the groups of Ree and Suzuki type. As we have already pointed out, it is not possible to find a reductive monoid $M$ with $0 \in M$ and $\operatorname{dim} Z G=1$ such that $\sigma: G \rightarrow G$ extends to a finite morphism $\sigma: M \rightarrow M$. 
So assume $G$ is an algebraic group of type $C_{2}, F_{4}$ or $G_{2}$ and $\sigma: G \rightarrow G$ is an endomorphism of Ree or Suzuki type. Assuming $\sigma(T)=T$ and $\sigma(B)=B$, with $T \subseteq B$, we can arrange the simple roots $\Delta=\left\{\alpha_{i}, \beta_{i}\right\}_{i=1}^{s}(s=1$ or 2) so that $\sigma^{*}\left(\alpha_{i}\right)=p^{a} \beta_{i}$ and $\sigma^{*}\left(\beta_{i}\right)=p^{a+1} \alpha_{i}$. (See $[19,11.6]$ for more details.) Choose $\mu=\sum_{i=1}^{2 s} a_{i} \lambda_{i}$, a dominant weight so that $a_{i}>0$ for $i>0$ and $a \lambda_{i} \in X(T)$ for all $i>0$. Then $\sigma^{*}(\mu)$ is also a dominant weight, and $\sigma^{*}(\mu)$ is not a multiple of $\mu$ since $\sigma^{*}$ has no rational eigenvalues on $X(T) \otimes \mathbb{R}$. Let $\bar{\mu}=\sigma^{*}(\mu) / p^{a} \in X(T)$. Let $\theta_{1}: G \rightarrow G l(V)$ and $\theta_{2}: G \rightarrow G l(W)$ be the irreducible representation of high weight $\mu$ and $\bar{\mu}$ respectively, and let $\rho=\theta_{1} \oplus \theta_{2}$. Define

$$
M_{1}=\overline{\rho(G)\left(k^{*} \times k^{*}\right)} \subseteq \operatorname{End}(V \oplus W)
$$

and let $M$ be the normalization of $M_{1}$. Using 6.3 and 6.4 of [15] and 4.1 of [14] it follows that if $\bar{T} \subseteq M$ is the closure of a maximal torus then $X(\bar{T})$ is the smallest polyhedral cone in $X(T)=X\left(T_{0}\right) \oplus \mathbb{Z}^{2}$ containing $W \cdot \xi \cup W \cdot \bar{\chi}$ where $\chi=(\mu, 1,0)$ and $\bar{\chi}=(\bar{\mu}, 0,1)$. The morphism $\sigma: G_{0} \rightarrow G_{0}$ extends to $\sigma: G \rightarrow G$ via $\sigma(g, s, t)=\left(\sigma(g), t^{p^{a+1}}, s^{p^{a}}\right)$ and this yields (by duality) $\sigma: T \rightarrow T$ via $\sigma^{*}(\gamma, \alpha, \beta)=\left(\sigma^{*} \gamma, p^{a+1} \beta, p^{a} \alpha\right)$. Since $\sigma^{*}(\chi)=p^{a} \bar{\chi}$ and $\sigma^{*}(\bar{\chi})=p^{a+1} \chi, \sigma^{*}(X(\bar{T})) \subseteq X(\bar{T})$ with $p^{2 a+1} X(\bar{T}) \subseteq \sigma^{*}(X(\bar{T}))$. Thus, $\sigma$ : $T \rightarrow T$ extends to $\sigma: \bar{T} \rightarrow \bar{T}$. Hence, by 4.5 of [15], there exists a unique $\sigma: M \rightarrow M$ extending $\sigma: \bar{T} \rightarrow \bar{T}$ and $\sigma: G \rightarrow G$. By Corollary 4.2 above $\sigma$ is a finite morphism.

By Corollary 3.2 of [15] it follows that $\Lambda$ has the following properties:

(1) $\Lambda_{1}=\left\{e_{1}, e_{2}\right\}$ and $\sigma\left(e_{1}\right)=e_{2}, \sigma\left(e_{2}\right)=e_{1}$.

(2) If $e \in \Lambda_{1}$, then $C_{G}(e)=T$ a maximal torus.

(3) $\Lambda \backslash\{0\}=\left\{f \in E(\bar{T}) \mid f e_{1}=e_{1}\right.$ or $\left.f e_{2}=e_{2}\right\}$.

Here $\Lambda_{1}$ consists of the minimal nonzero elements of $\Lambda$. Similarly $\Lambda_{2}$ consists of those elements of $\Lambda$ covering some element of $\Lambda_{1}$, etc. The sets $E_{i}=$ $E_{i}(M), i=1,2, \ldots$, are similarly defined.

Proposition 4.8. Let $\Lambda \subseteq M$ be a cross section lattice such that $\sigma(\Lambda) \subseteq \Lambda$. Then $\Lambda_{\sigma} \backslash\{0\}=\{e \in \Lambda \mid \sigma(e)=e, e \neq 0\}$ has a unique nonzero minimal element $e_{0}$. Further, $e_{0} \in \Lambda_{2}$.

Proof. Let $\Lambda_{1}=\left\{e_{1}, e_{2}\right\}$. It suffices to show that $e_{1} \vee e_{2} \in \Lambda_{2}$ since $\sigma\left(e_{1} \vee e_{2}\right)=$ $\sigma\left(e_{1}\right) \vee \sigma\left(e_{2}\right)=e_{2} \vee e_{1}=e_{1} \vee e_{2}$, and if $e \in \Lambda_{\sigma} \backslash\{0\}$ then $e>e_{1}$ or $e>e_{2}$. In the former case $e=\sigma(e)>\sigma\left(e_{1}\right)=e_{2}$ and so $e>e_{1} \vee e_{2}$.

Consider $\Lambda_{2}^{e_{1}}=\left\{f \in E(\bar{T}) \mid f\right.$ covers $\left.e_{1}\right\} \subseteq \Lambda$. Then $\left|\Lambda_{2}^{e_{1}}\right| \geq r k_{s s} G+1$ since $\operatorname{dim} T_{e_{1}}=r k_{s s} G+1$. Further, each $f \in \Lambda_{2}^{e_{1}}, f \neq e_{1} \vee e_{2}$ is of the form $f=e_{1} \vee e_{f}$ for some unique $e_{f} \in E_{1}(\bar{T})$. Notice that if $e_{f} \neq e_{2}$ then $e_{f}$ is $W$ conjugate to $e_{1}$ since otherwise there exists $\tau \in W$ such that $\left(e_{1} \vee e_{f}\right)^{\tau}=e_{2} \vee e_{1}^{\tau}$. But then $e_{2} \vee e_{1}^{\tau} \in \Lambda_{2}$, a contradiction. Thus, either $f=e_{1} \vee e_{2}$ or $f=e_{1} \vee e_{1}^{\tau}$ for some $\tau \in W$.

Since $\operatorname{dim} Z G=2, E\left(\overline{Z(G)^{0}}\right)=\{1, e, f, 0\}$ by [10, Remark 8.8]. So consider

$$
\varphi: M \rightarrow e M \times f M, \quad \varphi(x)=(e x, f x) .
$$

Then $\sigma(e)=f$, so $e>e_{1}$ and $f>e_{2}$ (or vice versa) and so $\varphi$ is a finite morphism. Also $e e_{2}=0=f e_{1}$ since $e f=0$. Notice that $e M e$ and $f M f$ 
are $\mathscr{J}$-irreducible with $J_{0}=\varnothing$ [13]. So let $h=e_{1} \vee e_{1}^{\tau} \in \Lambda_{2}^{e_{1}}$. Then $e h=h$ and $f h=0$ since $e E_{1}(M)=C l_{G}\left(e_{1}\right)$ and $f E_{1}(M)=C l_{G}\left(e_{2}\right)$ and $e f=0$. Since $e M e$ is $\mathscr{J}$-irreducible with $J_{0}=\varnothing$ there exists a unique $\alpha \in \Delta$ such that $\sigma_{\alpha} h \sigma_{\alpha}=h$ (4.13 of [13]). Thus $e_{1}^{\tau}=e^{\sigma_{2}}$ and so $\tau=\sigma_{\alpha}$. Therefore

$$
\Lambda_{2}^{e_{1}} \subseteq\left\{e_{1} \vee e_{2}, e_{1} \vee e_{1}^{\sigma_{\alpha_{1}}}, \ldots, e_{1} \vee e_{1}^{\sigma_{\alpha_{r}}}\right\}
$$

where $r=r k_{s s} G$. But from before, $\left|\Lambda_{2}^{e_{1}}\right| \geq r k_{s s} G+1$. Thus $e_{1} \vee e_{2} \in \Lambda_{2}^{e_{1}}$.

Proposition 4.9. Let $e \in E(\bar{T})_{\sigma}, e \neq 0$. Then $e=g_{1} \vee \cdots \vee g_{t}$ where each $g_{i}$ is $W$-conjugate to $e_{0}=e_{1} \vee e_{2}$.

Proof. First note that $e \in \Lambda_{2 i}$ for some $i \geq 0$, since $X(T)$ has no $\sigma^{*}$-invariant subgroup of odd rank. If $G$ is of type $C_{2}$ or $G_{2}$ then by Proposition 4.8, $\Lambda_{\sigma}=\left\{1, e_{1} \vee e_{2}, 0\right\}$ and $W_{\sigma}=\{1, s\}$ with $\left(e_{1} \vee e_{2}\right)^{s} \neq e_{1} \vee e_{2}$. If $G$ is of type $F_{4}$ then $\Lambda_{\sigma}=\{0\} \cup\left\{e_{1} \vee e_{2}\right\} \cup\left(\Lambda_{4}\right)_{\sigma} \cup\{1\}$. So it suffices to show that any $e \in\left(\Lambda_{4}\right)_{\sigma}$ satisfies the claim, since the other cases are obvious. For this it suffices to show that there are at least two elements in the set $\left\{f \in E_{2}(\bar{T}) \mid f \leq\right.$ $e, \sigma(f)=f\}$, since these idempotents are all conjugate. Consider $e M e$. Then $\mathscr{U}(e M e)=e \Lambda$ and $\sigma$ acts on $e \Lambda$. $e M e$ is not a $D$-monoid since its rank is four while $\left|(e \Lambda)_{1}\right|=2$. Thus, there exists $w \in \Lambda_{\sigma}$ such that $w e=e w \neq e$. But if $e \geq e_{1} \vee e_{2}$ then $e=e^{w} \geq\left(e_{1} \vee e_{2}\right)^{w}$. But $C_{W}\left(e_{1} \vee e_{2}\right)=\{1\}$ and so $e_{1} \vee e_{2} \neq\left(e_{1} \vee e_{2}\right)^{w}$.

Theorem 4.10. Let $M_{\sigma}=\{x \in M \mid \sigma(x)=x\}$ where $M_{\sigma}$ is as above. Let $\Gamma$ be the set of simple reflections of $W_{\sigma}$ relative to $B_{\sigma}$. Then $M_{\sigma}$ satisfies the following properties:

(a) $\Lambda_{\sigma} \backslash\{0\} \cong 2^{\Gamma}$ via $e \mapsto I(e), C_{G_{\sigma}}^{r}(e)=P_{I(e)}$.

(b) If $I(e)=I, I(f)=K \subseteq \Gamma$ let $J_{I}=G_{\sigma} e G_{\sigma}$ and $J_{K}=G_{\sigma} f G_{\sigma}$. Then $J_{I} J_{K} \subseteq J_{I \cap K} \cup\{0\}$.

Proof. The proof follows formally from what has been written down in [13], once a few observations are made. 4.3 of [13] is true of $M_{\sigma}$ by $1.3,1.10$ above and [11, Theorem 3.3]. 4.4 of [13] with $\Lambda_{\sigma}$ in place of $\Lambda$ follows from the fact that if $e \in \Lambda_{\sigma}$ then $(e M e, \sigma)$ is a monoid of the same type as $(M, \sigma)$, and hence has no central $\sigma$-stable idempotents $\neq 0,1.4 .5$ is true vacuously since $J(e)=\varnothing$ for all $e \in \Lambda \backslash\{0\}$. 4.6 and 4.12 follow from 4.3 and $J(e)=\varnothing$. Finally 4.13 follows routinely. In our case it states the following: Let $e \in \Lambda_{\sigma} \backslash\{0\}$. Then there is a one-to-one correspondence between $\left\{\sigma_{\alpha} \in S \mid \sigma_{\alpha} e \neq e \sigma_{\alpha}\right\}$ and $\left\{f \in \Lambda_{\sigma} \mid f\right.$ covers $\left.e\right\} . \sigma_{\alpha}$ corresponds to the unique $f$ such that $C_{G_{\sigma}}^{r}(f)=P_{I(e) \cup\left\{\sigma_{\alpha}\right\}}$.

Starting with $e_{0}=e_{1} \vee e_{2}$ and applying this to $\Lambda_{\sigma}$ yields part (a). For part (b), notice that the claim is valid for $M$, while if $x \in M_{\sigma}$ then, as before, $G x G \cap M_{\sigma}=G_{\sigma} x G_{\sigma}$.

There are many finite monoids having the properties of Theorems 4.3, 4.7, 4.10. The abstract universal monoids $\mathscr{M}$ constructed in $\S 1$ are such that the unit groups $H_{e}$ of each $e \mathscr{M} e, e \in E(\mathscr{M})$ are as large as possible. In the monoids $M_{\sigma}$ above, a local group $H_{e}$, instead of being isomorphic to some Levi subgroup $L$ of $G\left(M_{\sigma}\right)$ is instead isomorphic to $L / N$ for some central subgroup $N$ of $L$. This creates problems in relating the representation theories of $G_{\sigma}$ and $M_{\sigma}$. We are therefore naturally led to considering the universal central extension $\mathscr{M}$ of $M_{\sigma}$. 
Finally the authors would like to thank the referee for useful suggestions.

\section{REFERENCES}

1. R. W. Carter, Finite groups of Lie type: Conjugacy classes and complex character, Wiley, 1985.

2. A. H. Clifford, Matrix representations of completely simple semigroups, Amer. J. Math. 64 (1942), 327-342.

3. _ Basic representations of completely simple semigroups, Amer. J. Math. 82 (1960), 430-434.

4. A. H. Clifford and G. B. Preston, Algebraic theory of semigroups, Vol. 1, Math. Surveys No. 7, Amer. Math. Soc., Providence, R.I., 1961.

5. C. W. Curtis, Modular representations of finite groups with split $(B, N)$-pair, Lecture Notes in Math., vol. 131, Springer-Verlag, 1970, pp. 57-95.

6. C. DeConcini and C. Procesi, Complete symmetric varieties, Lecture Notes in Math., vol. 996, Springer-Verlag, 1984, pp. 1-44.

7. J. C. Harris and N. J. Kuhn, Stable decomposition of classifying spaces of finite abelian p-groups, Math. Proc. Cambridge Philos. Soc. 103 (1988), 427-449.

8. J. Okniński and M. S. Putcha, Complex representations of matrix semigroups, Trans. Amer. Math. Soc. (to appear).

9. M. S. Putcha, A semigroup approach to linear algebraic groups, J. Algebra 80 (1983), 164185.

10. __ Linear algebraic monoids, London Math. Soc. Lecture Note Series 133, Cambridge Univ. Press, 1988.

11. __ Monoids on groups with BN-pairs, J. Algebra 12 (1989), 139-169.

12. __ Monoids of Lie type and group representations, Monoids and Semigroups with Applications (J. Rhodes, ed.), World Scientific, 1991, pp. 288-305.

13. M. S. Putcha and L. E. Renner, The system of idempotents and the lattice of $\mathscr{J}$-classes of reductive algebraic monoids, J. Algebra 116 (1988), 385-399.

14. L. E. Renner, Classification of semisimple rank one monoids, Trans. Amer. Math. Soc. 287 (1985), 457-473.

15. _ Classification of semisimple algebraic monoids, Trans. Amer. Math. Soc. 292 (1985), 193-223.

16. __ Analogue of the Bruhat decomposition for algebraic monoids, J. Algebra 101 (1986), 303-338.

17. __ Finite monoids of Lie type, Monoids and Semigroups with Applications (J. Rhodes, ed.), World Scientific, 1991, pp. 278-287.

18. L. Solomon, The Bruhat decomposition, Tits system and Iwahori ring for the monoid of matrices over a finite field, Geom. Dedicata 36 (1990), 15-49.

19. T. A. Springer and R. Steinberg, Conjugacy classes, Lecture Notes in Math., vol. 131, Springer-Verlag, 1970, pp. 57-95.

20. R. Steinberg, Endomorphisms of linear algebraic groups, Mem. Amer. Math. Soc. 80 (1968).

Department of Mathematics, North Carolina State University, Raleigh, North CarOLINA 27695-8205

Department of Mathematics, University of Western Ontario, London, Ontario, Canada N6A 5B7 